\title{
Global Connecting Dual Representation of Non-Smooth Multi-Objective Programs using Convex Functions
}

\author{
Jeyalakshmi P.
}

\begin{abstract}
New classes of vector functions, namely $V-(b, F, \rho)-$ pseudo convex and $V-(b, F, \rho)$ - quasi convex functions are introduced which are weaker than $V-(b, F, \rho)$ - convex functions. Sufficient optimality conditions for proper potency and numerous duality theorems for a category of nonsmooth multiobjective programs are obtained under the assumptions of the above said functions.
\end{abstract}

Keywords: Duality, Nonsmooth multiobjective programs, Optimality, $V-(b, F, \rho)-$ pseudo convex and $V-(b, F$, p)-quasiconvex.

\section{INTRODUCTION}

$\mathrm{E}_{\text {gudo and Hanson [2] extensive the ideas of V-invexity }}$ of Jeyakumar and Mond [5] to nonsmooth vector functions. Also, they established ample optimality criteria for potency and Wolfe kind duality results for a category of nonsmooth multiobjective programs beneath the assumptions of V-invexity Mishra and Mukherjee[8] extended V -pseudo invexity and V-quasi invexity of Jeyakumar and Mond [5] to nonsmooth vector functions. Further, they verified adequate optimality conditions for potency and Mond-Weir tupe duality results for a category of nonsmooth multiobjective programs underneath the assumptions of the higher than same functions Lalitha [6] introduced the nonsmooth vector functions ,namely, $\mathrm{V}$-b-invex , $\mathrm{V}$-pseudo b-invex and V-quasi $\mathrm{b}$-invex functions as a generalization of $\mathrm{V}$-invex functions of Egudo and Hanson[2] and V-pseudo invex and V-quasi invex functions of Mishra and Mukherjee[8] severally. Also, she studied the interrelationship among V-b-invex V-pseudo b-invex, V-guasi b-invex functions. Further, she used the on top of aforementioned functions to get optimality conditions for potency and duality consequences for nonsmooth multiobjective programs connecting Lipschitz functions. Recently, Pandian[9] has introduced a replacement category of nonsmooth vector functions particularly, V-(b,F, $\rho)$-convex functions as a generalization of V-b-invex functions. Then ,he establish spare optimality conditions for correct potency and varied duality theorems for multiobjective programs connecting Lipschitz functions underneath the assumptions of

Revised Manuscript Received on December 15, 2019.

* Correspondence Author

P. Jeyalakshmi*, Department of Mathematics, Kalasalingam Academy of Research and Education, Krishnankovil, Tamilnadu. Email: jeyalakshmi.p@klu.ac.in the higher than aforesaid functions

This article, we initiate new categories of nonsmooth vector functions, that is to say $V-(b, F, \rho)$ - pseudo convex and $V-(b, F, \rho)$-quasiconvex functions as a generalization of $\mathrm{V}$-pseudo $\mathrm{b}$-invex and $\mathrm{V}$-quasi b-invex functions of Lalitha [6] respectively and also, we prove that they are weaker than $V-(b, F, \rho)$-convex functions introduced by Pandian[9]. Further, we start adequate optimality conditions for correct potency and varied duality theorems for nonsmooth multiobjective programs connecting Lipschitz functions beneath the assumptions of the above said functions.

\section{PRELIMINARIES}

Let $\mathrm{X}$ be a nonempty open broken-backed set of $R^{n}$ and $R_{+}$be the set of all non negative real numbers.

Throughout this paper, the following Mangasarian's [7] conventions for vectors in $R^{n}$. Then

$x=y$ if and only if $x_{i}=y_{i}$ for all i;

$x \leq y$ if and only if $x_{i} \leq y_{i}$ for all $\mathrm{i}$;

$x_{r} \prec y_{r}$ is the negation of $x \leq y$.

Also, we use the following notation in this paper.

If $\alpha=\left(\alpha_{1}, \ldots, \alpha_{n}\right)$ and $\beta=\left(\beta_{1}, \ldots, \beta_{n}\right)$

then $\alpha \beta=\left(\alpha_{1} \beta_{1}, \ldots, \alpha_{n} \beta_{n}\right)$.

We the following definitions which can be found in Clarke [1]

Definition 2.1. A function $h: X \rightarrow R$ is Lipschitz on $\mathrm{X}$ if there exists a relentless $\mathrm{K}$ such for all $x_{s} y \in X_{v} \quad|h(x)-h(y)| \leq\|x-\|$

Definition 2.2. If $h: X \rightarrow R$ is Lipschitz on $\mathrm{X}$, the generalized directional by-product of h at $x \in X$ within the direction $v \in R^{n}$, denoted by $h^{*}(x ; v)$ is given by

$$
h^{*}\left(x_{*}^{*} v\right)=\lim _{\substack{y \rightarrow x \\ d \downarrow 0}}\left[\frac{h(y+d v)-h(y)}{d}\right]
$$

Definition 2. 3. The generalized gradient of $h: X \rightarrow R$ at $x \in X$, denoted by $\partial h(x)$ is outlined as follows 
$\partial h(x)=\left\{\xi \in R^{n} h^{0}(x: v) \geq \xi^{t} v_{x}\right.$ for all $\left.v \in R^{n}\right\}$

The following definition of the sub linearity can be in Hanson and Mond [7].

Definition 2.4. A functional $F: X x X x R \rightarrow R \quad$ is said to be sublinear on $R^{n}$ if for any $x, y \in X$,

$F(x, u ; a+b) \leq F(x, u ; a)+F(x, u ; b)$ for $a l l a, b \in R^{n}$ and

$F(x, u, t a)=t F(x, u, a)$, for all $a \in R^{n}$

and for all $t \geq 0$ in $\mathrm{R}$

Note 2.1. $F(x, u ; 0)=0$ for all $x, y \in X$

Let $f=\left(f_{1}, . ., f_{k}\right)$ and $g=\left(g_{1}, . ., g_{m}\right)$ be Lipscitz functions on $\mathrm{X}$ where $f_{i}$ and $f_{i}: X \rightarrow R$, for all $\mathrm{i}$ and $\mathrm{j}$, $\partial f_{i}(u)$ denote the generalized subgradient of $f_{i}$ at $u$, $b=\left(b_{1, \ldots,}, b_{k}\right)$ and $b=\left(c_{1, \ldots,} c_{\mathrm{m}}\right)$

where $b_{i}$ and $c_{j}: X x X \rightarrow R, \quad$ for all $\mathrm{i}$ and $\mathrm{j}$, $\rho=\left(\rho_{1, \ldots \ldots} \rho_{k}\right)$ and $\gamma=\left(\gamma_{1, \ldots x} \gamma_{m}\right)$ where $\rho_{i}$ and $\gamma_{j} \in R \quad$,for $\quad$ all $\quad \mathrm{i} \quad$ and $\quad \mathrm{j} \quad \eta: \eta x \eta \rightarrow R^{n}$,

$F: X x X x R^{n} \rightarrow R$ be sublinear on $R^{n}$ and $d: X x X \rightarrow R$ such that $d(x, x)=0$ for all $x \in X$.

Consider the subsequent nonsmooth multiobjective programming downside $(\mathrm{P})$,

(P) Minimize $f(x)$

$$
\text { Subject to } g(x) \leq 0, x \in X \text {. }
$$

In connection to $(\mathrm{P}), \quad$ we consider the following Mond-Weir type dual nonsmooth multiobjective

(D) Maximize $f(u)$

$$
0 \in \sum_{i=1}^{\substack{\text { Subject to } \\ k}} \lambda_{i} \partial f_{i}(u)+\sum_{j=1}^{m} \mu_{j} \partial g_{j}(u)
$$

$\mu_{j} g_{j}(u) \geq 0$ for all $\mathrm{j}$,

$\lambda>0, \mu \geq 0$ and $\lambda^{t} e=1$

Where $\quad u \in X, \lambda=\left(\lambda_{1}, \ldots, \lambda_{k}\right)$ in $R^{k}$ $\mu=\left(\mu_{1}, \ldots, \mu_{m}\right)$ in $R^{m}$ and $e=(1, \ldots, 1) \in R^{k}$

Let $\mathrm{S}$ be the set of all probable solutions of the matter $(\mathrm{P})$ and alphabetic character be the set of all feasible solutions of the problem

We need the subsequent definitions and lemma which may be found in Geoffrion [3].

Definition 2.5. A possible purpose $x^{0}$ of $(\mathrm{P})$ is alleged to be associate economical resolution of $(\mathrm{P})$ if exists no different feasible point $\mathrm{x}$ of $(\mathrm{P})$ such $f(x) \leq f\left(x^{0}\right)$

Definition 2.6. An efficient solution $x^{0}$ of $(\mathrm{P})$ is said to

$M \succ 0$ such that for each i for each feasible point x of (P) for which $f_{i}(x)-f_{i}\left(x^{0}\right) \prec 0$,

we have

$f_{i}(x)-f_{i}\left(x^{0}\right) \leq M\left(f_{i}(x)-f_{i}\left(x^{0}\right)\right)$, programming problem (D). be property efficient solution of $(\mathrm{P})$ if there exists a scalar

for some $\mathrm{r}$ such that $f_{r}(x)-f_{r}\left(x^{0}\right) \succ 0$.

Lemma 2.1. Let $\lambda^{t} \succ 0$ in $R^{k}$ with $\lambda^{0 t} e=1$ be fixed. If $x^{0}$ is an optimal solution for the scalar programming problem $\left(P_{X}\right)$,

$\left(P_{X}\right), \quad$ Minimize $\lambda^{0 t} f(x), x \in S$,

then $x^{0}$ is a properly well-organized answer for $(\mathrm{P})$.

We need the following necessary optimality theorem for efficiency for proving the strong duality theorem which can be found in Lalitha[3].

Theorem2.1. Let $x^{0}$ be an well-organized answer for $(\mathrm{P})$ at which a restraint requirement [7] is contented for each scalar program $\left(P_{t}\left(x^{0}\right)\right), \mathrm{t}=1,2, \ldots, \mathrm{k}$ where

$$
\begin{aligned}
& \left(P_{t}\left(x^{0}\right)\right) \text { Minimize } f_{t}(x) \\
& \quad \text { Subject to } \\
& \quad f_{i}(x) \leq f_{i}\left(x^{0}\right), \text { for all i } i \neq t, x \in S .
\end{aligned}
$$

Then there exists $\lambda^{0} \succ 0$ in $R^{k}$ with $\lambda^{0 t} e=1$ and $\mu^{0} \geq 0$ in $R^{m} \quad$ such that the following conditions hold

$$
0 \in \sum_{i=1}^{k} \lambda_{i}^{0} \partial f_{i}\left(x^{0}\right)+\sum_{j=1}^{m} \mu_{j}^{0} \partial g_{j}\left(x^{0}\right)
$$

$$
\mu_{j}^{0} \partial g_{j}\left(x^{0}\right)=0 \text {, for all } \mathrm{j}, \mathrm{j}=1,2, \ldots, \mathrm{m} \text {. }
$$

We require the next definitions and results which can be found in Lalitha[3].

We need the following definition of convex and the result which can be found in Pandian [9].

Definition 2.7. The function $f$ is said to be $V-(b, F, \rho)$ - convex at $u \in X$ if for all $x \in X \quad$ and for all $\xi_{\mathrm{i}} \in \partial f_{\mathrm{i}}(u)$.

$b_{i}(x, u)\left[f_{i}(x)-f_{i}(u)\right] \geq F(x, u)$ for all $i$

Remark 2.1. Every V-b- invex function is $V-(b, F, \rho)$ - convex function by taking $F(x, u ; z)=z^{t} \eta(x, w)$

and $\rho=0$, but the converse is not true.

\section{III . GENERALIZED $V-(b, F, \rho)$ - CONVEX FUNCTIONS.}

We, now define new classes of vector functions, namely $V-(b, F, \rho)$ - quasi convex and $V-(b, F, \rho)$ pseudo convex functions.

Definition 3.1. The function $\mathrm{f}$ is aforementioned to be $V-(b, F, \rho)$ - quasi convex at $u \in X$ if for all $x \in X$ and for all $\quad \xi_{\mathrm{i}} \in \partial f_{\mathrm{i}}(u)_{x}$,

$$
\sum_{i=1}^{k} b_{i}(x, u) f_{i}(x) \leq \sum_{i=1}^{k} b_{i}(x, u) f_{i}(u) \Rightarrow \sum_{i=1}^{k} F\left(x, u ; \xi_{i}\right)+\sum_{i=1}^{k} p_{i} d^{2}(x, u) \leq 0 .
$$

If in the above definition, ( 3 is satisfied as 
$\sum_{i=1}^{k} b_{i}(x, u) f_{i}(x) \leq \sum_{i=1}^{k} b_{i}(x, u) f_{i}(u) \Rightarrow \sum_{i=1}^{k} F\left(x, u ; \xi_{i}\right)+\sum_{i=1}^{k} p_{i} d^{2}(x, u)<0$,

Then we say that $f_{\text {is }} \mathrm{V}$-properly $\left(\boldsymbol{b}_{v} \boldsymbol{F}, \boldsymbol{\rho}\right)$ _ quasi convex at $\mathrm{u}$.

If in the above definition, $x \neq u$ and (3) is satisfied as $\sum_{i=1}^{k} b_{i}(x, u) f_{i}(x) \leq \sum_{i=1}^{k} b_{i}(x, u) f_{i}(u) \Rightarrow \sum_{i=1}^{k} F\left(x, u ; \xi_{i}\right)+\sum_{i=1}^{k} \rho_{i}$

Then we say that $f$ is $\mathrm{V}$-strictly $-(b, F, \rho)$ quasi convex at $\mathrm{u}$.

Remark 3.1. Every V-quasi b-invex function with respect to $b(x, u)$ and $\eta(x, u)$ is $V-(b, F, \rho)$ quasi convex by taking $\quad F(x, u ; z)=z^{t} \eta(x, u)$ and $\rho=0$,but the converse is not true. This is proved by the following example.

Example 3.1. Let $X=(-1,1), f=\left(f_{1}, f_{2}\right), b=\left(b_{1}, b_{2}\right)$ and $\quad \boldsymbol{\rho}=\left(\boldsymbol{P}_{1}, \boldsymbol{\rho}_{2}\right)=(\mathbf{- 4},-\mathbf{1})$. Define the functions $f_{1}$ and $f_{2}: X \rightarrow R_{x} \quad b_{1}$ and $\quad b_{2}: X x X \rightarrow R_{+} F: X x X x R^{2} \rightarrow R$ and d: $X x X \rightarrow R$ as follows.

$$
\begin{aligned}
& f_{1}(x)=\left\{\begin{array}{cc}
x+1 & : x \geq 0 \\
1 & : x \leq 0
\end{array}, \quad f_{2}(x)=\left\{\begin{array}{cc}
2 x+1: x \geq 0 \\
1 \quad: x \leq 0
\end{array}\right.\right. \\
& b_{1}(x, u)=\left\{\begin{array}{cc}
1: & : \neq \neq 0 \\
0 & : u=0,
\end{array}, \quad b_{2}(x, u)=\left\{\begin{array}{cc}
1: u \neq 0 \\
0: u=0 .
\end{array}\right.\right. \\
& F(x, u ; z)=z(x-u)^{2}, \text { and } d(x, u)=\sqrt{\left|x^{2}-u^{2}\right|} \text {. Then }
\end{aligned}
$$

$f$ is namely $V-(b, F, \rho)$ - quasi convex at $\mathrm{u}=0$, but not $\mathrm{V}$-quasi b-invex at $u=0$ by means of value to $\eta(x, u)=(x-u)^{2}$, for $\quad x=\frac{1}{2} \xi_{1}=\frac{1}{2}$ and $\xi_{2}=\frac{a}{4}$

$\sum_{\mathrm{i}=1}^{2} b_{\mathrm{i}}(x, u) f_{\mathrm{i}}(x) \leq \sum_{\mathrm{i}=1}^{2} b_{\mathrm{i}}(\mathrm{x}, \mathrm{u}) \mathrm{f}_{\mathrm{i}}(\mathrm{u})$

and

$\sum_{i=1}^{2} \xi_{j}^{t} \eta(x, u)>0$.

Remark 3.2. Every $V-(b, F, \rho)$ - convex function is $V-(b, F, \rho)-$ quasi convex ,but the converse is not true.

Definition 3.2. The function $\mathrm{f}$ is said to be $V-(b, F, \rho)$ pseudo convex at $u \in X$ if for all $x \in X$ and for all

$$
\xi_{i} \in \partial f_{i}(u) \text {. }
$$

$\sum_{i=1}^{k} F\left(x, u_{i}, \xi_{i}\right)+\sum_{i=1}^{k} p_{i} d^{2}(x, u) \geq 0$,

$\Rightarrow \sum_{i=1}^{k} b_{i}(x, w) f_{i}(x) \leq \sum_{i=1}^{k} b_{i}(x, w) f_{i}(u)$

In the above defiition (4) is satisfied as $\sum_{i=1}^{k} F\left(x, u_{i}, \xi_{i}\right)+\sum_{i=1}^{k} \rho_{i} d^{2}(x, u) \geq 0$,

$$
\Rightarrow \sum_{i=1}^{k} b_{i}(x, u) f_{i}(x) \leq \sum_{i=1}^{k} b_{i}(x, w) f_{i}(u)
$$

Then we say that $\mathrm{f}$ is $\mathrm{V}$-properly $-(b, F, \rho)$ pseudo convex at $\mathrm{u}$.

If in the over definition, and $x \neq u$

(4) is satisfied as

$\sum_{i=1}^{k} F\left(x, u_{i}, \xi_{i}\right)+\sum_{i=1}^{k} p_{i} d^{2}(x, w) \geq 0$,

$\Rightarrow \sum_{i=1}^{k} b_{i}(x, w) f_{i}(x) \leq \sum_{i=1}^{k} b_{i}(x, w) f_{i}(u)$

Then we state that $f$ is $\mathrm{V}$-strictly $-(b, F, \rho)$ pseudo convex

at $u$.

Remark 3.3. Every V-pseudo b-invex function by means of value to $b(x, u)$ and $\eta(x, u)$ is $V-(b, F, \rho)$ - pseudo convex by taking $F(x, u ; z)=z^{t} \eta(x, u)$ and $\rho=0$, but the converse is not true.

\section{SUFFICIENT OPTIMALITY CONDITIONS}

In this part we have a tendency to prove adequate optimality conditions for a possible purpose of $(\mathrm{P})$ to be a $\rho_{\mathrm{i}}$ properly <edonomical resolution for $(\mathrm{P})$ underneath the assumptions of $V-(b, F, \rho)$ - pseudo convexity and $V-(c, F, \gamma)$ quasi convexity on the functions involved.

Theorem 4.1. Let $x^{0} \in S$ and there exists scalars $\lambda^{0} \succ 0 \quad$ in $\mathrm{R}^{\mathrm{n}}$ with $\sum_{\mathrm{i}=1}^{\mathrm{k}} \lambda_{\mathrm{i}}^{0}=1$ and $\mu_{\mathrm{j}}^{\mathrm{Q}} \geq 0$ in $\mathrm{R}$, for all $\mathrm{j}$ such that

$$
\begin{aligned}
& \left.0 \in \sum_{i=1}^{k} \lambda_{i}^{0} \partial f_{i}\left(x^{0}\right)+\sum_{j=1}^{m} \mu_{j}^{0} \partial g_{j}\left(x^{0}\right)\right) \\
& \mu_{j}^{0} \partial g_{j}\left(x^{0}\right)=0 \\
& \text { for all } \mathrm{j} \text {. }
\end{aligned}
$$

If $\lambda^{0} f$ is of $V-(b, F, \rho)$ - pseudo convex at $x^{0}$ with $b\left(x, x^{0}\right) \succ 0 \quad$ for $\quad$ all $\quad x \in S \quad . \quad \mu^{0} g \quad$ is $V-(c, F, \gamma)$-quasi convex at $x^{0}$ such that

$\sum_{i=1}^{k} \rho_{i}+\sum_{j=1}^{m} \gamma_{j} \quad \geq 0$

Then $x^{0}$ is a properly well-organized answer for $(\mathrm{P})$.

Proof: Let $x \in S$. Now from (5) and by the insurable of F, we have

$$
F\left(x_{x} x^{0,} \sum_{i=1}^{k} \lambda_{i}^{0} \alpha_{i}\right) \geq-F\left(x, x^{0}, \sum_{j=1}^{m} \mu_{j}^{0} \beta_{j}\right),
$$

for some $\alpha_{i} \in \partial f_{i}\left(x^{0}\right)$ for all $i$ and $\beta_{i} \in \partial g_{j}\left(x^{0}\right)$ for all $\mathrm{j}$. Now, since $x \in S$ and $c_{j}\left(x_{v} x^{0}\right) \geq 0$ and $\mu_{j}^{0} \geq 0$, for all $\mathrm{j}$ and from (6) and by the $V-(c, F, \gamma)$ - quasi convex at $\mu^{0} g$ and by the insurable of $\mathrm{F}$,

$$
F\left(x, x^{0}{ }_{j} \sum_{j=1}^{m} \mu_{j}^{0} \beta_{j}\right)+\left(\sum_{j=1}^{\mathrm{m}} \gamma_{j}\right) d^{2}\left(x, x^{0}\right) \leq 0
$$

Now , from (7), (8) and (9), we get

$F\left(x_{*} x^{0} \sum_{i=1}^{\mathbb{k}} \lambda_{i}^{0} \alpha_{i}\right)+\left(\sum_{i=1}^{\mathbb{k}} \rho_{i}\right) d^{2}\left(x, x^{0}\right) \geq 0$

By the sublinearity of $\mathrm{F} V-(b, F, \rho)$-quasi convex at $\lambda^{0} f$ it follows that

$$
\left.0 \in \sum_{i=1}^{k} \lambda_{i}^{0} \partial f_{i}\left(x^{0}\right)+\sum_{j=1}^{m} \mu_{j}^{0} \partial g_{j}\left(x^{0}\right)\right)
$$

for all $x \in S$ -

Suppose that $x^{0}$ is not well-organized for $(\mathrm{P})$. Then , there exists $w \in S$ such that $f(w) \leq f\left(x^{0}\right)$. 
Since $b\left(w x^{0}\right)>0$

And $\lambda^{0}>0$ with $\lambda^{\text {ot } e}=1$, we can conclude that

$\sum_{\mathrm{i}=1}^{k} b_{\mathrm{i}}\left(w, x^{0}\right) \lambda_{\mathrm{i}}^{0} f_{\mathrm{i}}(w)<\sum_{\mathrm{i}=1}^{k} b_{\mathrm{i}}\left(w, x^{0}\right) \lambda_{\mathrm{i}}^{0} f_{\mathrm{i}}\left(x^{0}\right)$

which contradicts (10). Thus is $x^{\mathbb{0}}$ an well-organized answer for $(\mathrm{P})$.

Suppose that $x^{0}$ is not a properly well-organized answer for (P) then, there exists a point $v \in S$ and an index i such that $b\left(w x^{0}\right)>0$

And $\lambda^{0}>0$ with $\lambda^{\text {ot }} e=1$, we have

$\sum_{i=1}^{k} b_{i}\left(v_{v} x^{0}\right) \lambda_{i}^{0} f_{i}(v)<\sum_{i=1}^{k} b_{i}\left(v_{v} x^{0}\right) \lambda_{i}^{0} f_{i}\left(x^{0}\right)$

which contradicts (10). Thus is $x^{0}$ is a proper efficient solution for $(\mathrm{P})$.

Remark 4.1. The over Theorem 1 can be proved in a similar manner under any one of the subsequent assumptions

(i) $\lambda^{0} f \quad$ is V-properly $-(b, F, \rho)$ pseudoconvex at $x^{0}$ and $\mu^{0} g$ is $V-(c, F, \gamma)$ quasi convex at $x^{0}$ such that (7) is satisfied.

(ii) $\quad \lambda^{0} f$ is $\mathrm{V}$-properly $-(b, F, \rho)$ quasi convex at $x^{0}$ and $\mu^{0} g$ is $V-(c, F, \gamma)$ quasi convex at $x^{0}$ such that (7) is satisfied.

\section{DUALITY THEOREMS}

We, currently get varied duality theorems between $(\mathrm{P})$ and (D) underneath the assumptions of $V-(b, F, \rho)$-pseudo convexity and $V-(c, F, \gamma)$ - quasi convexity on the functions concerned.

Theorem 5.1. (Weak Duality) . Let $x \in S$ and $\left(u, \lambda_{s} \mu\right) \in Q$. If $\lambda f$ is at $\mathrm{u}$ with $b(x, u)>0$, for all $x \in S$ and $\mu g$ is $V-(c, F, \gamma)$ - quasi convex at ${ }^{u}$ such that

$\sum_{i=1}^{k} \rho_{i}+\sum_{j=1}^{m} \gamma_{j} \geq 0$

(11)

then $f(x) \$ f(u)$.

Proof: Suppose that $f(x) \leq f(u)$. Since $\lambda>0$ with $\lambda^{t} e=1$ and $b(x, u)>0$ and by $\mathrm{V}_{-}\left(b, F_{s}, \rho\right)-$ pseudo convexity

of $\lambda f$ and the insurable of $\mathrm{F}$, it follows that

$F\left(x, u_{i} \sum_{i=1}^{k} \lambda_{i} \alpha_{i}+\left(\sum_{i=1}^{k} \rho_{i}\right) d^{2}(x, u)<0\right.$

Now, from (1) and by the insurable of $F$, we have

$$
F\left(x, u_{i} \sum_{i=1}^{k} \lambda_{i} \alpha_{i} \geq-F\left(x, u_{i} \sum_{j=1}^{m} \mu_{j} \beta_{j}\right)\right.
$$

$f_{i}\left(x^{0}\right)-f_{i}(v)$ can be made arbitrarily large and hence

for some $\alpha_{\mathrm{i}} \in \partial f_{\mathrm{i}}(u)_{x}$ for all $\mathrm{i}$ and $\beta_{j} \in \partial f_{\mathrm{i}}(u)_{x}$ for all $\mathrm{j}$. Now since $x \in S$ and $\left(u, \lambda_{s} \mu\right) \in Q$ and by the $V-(c, F, \gamma)$ - quasi convex at $\mu g$ and the insurable of $\mathrm{F}$, we have

$$
F\left(x, u_{i} \sum_{j=1}^{m} \mu_{j} \beta_{j}+\left(\sum_{j=1}^{m} \gamma_{j}\right) d^{2}(x, u) \leq 0\right.
$$

Now , from (11),(13) and ,(14), we get

$$
F\left(x, u_{i}^{*} \sum_{i=1}^{k} \lambda_{i} \alpha_{i}+\left(\sum_{i=1}^{k} \rho_{i}\right) d^{2}(x, u) \geq 0\right.
$$

which contradicts (12). Thus, $f(x) \$ f(u)$.

Theorem 5.2. (Well-built Duality) . Let $x^{0}$ be an well-organized answer for $(\mathrm{P})$ at which a restraint requirement [7] is content for each scalar program $\left(P_{t}\left(x^{0}\right)\right) \quad \mathrm{t}=1,2, \ldots, \mathrm{k}$ where

$$
\left(P_{t}\left(x^{0}\right)\right) \text {, Minimize } f_{t}(x)
$$

\section{Subject to}

$$
f_{i}(x) \leq f_{i}\left(x^{0}\right), \text {, for all i } i \neq t, x \in S .
$$

Then, there exists $\lambda^{0}>0$ in $R^{k}$ with $\lambda^{0 t} e=1$ and $\mu^{0} \geq 0$ in $R^{m}$ such that $\left(x^{0}, \lambda^{0}, \mu^{0}\right)$ is in Q. Further, if the feeble duality theorem 2 holds between ( P) and (D), then $\left(x^{0}, \lambda^{0}, \mu^{0}\right)$ is a properly well-organized answer for for (D).

Proof: Since $x^{0}$ is an well-organized answer for $(\mathrm{P})$ at which a restraint requirement is content for each scalar program, $\left(P_{t}\left(x^{0}\right)\right), t=1,2, \ldots, k$ and the Theorem 1 in Lalitha [6] , there exists $\lambda^{0}>0$ in $R^{k}$ with $\lambda^{\text {ot }} e=1$ and $\mu^{0} \geq 0$ in $R^{m}$ such that

$$
0 \in \sum_{i=1}^{k} \lambda_{i}^{0} \partial f_{i}\left(x^{0}\right)+\sum_{j=1}^{m} \mu_{j}^{0} \partial g_{j}\left(x^{0}\right)
$$

$\mu_{j}^{0} \partial g_{j}\left(x^{0}\right)=0$, for all $\mathrm{j}$

Thus $\left(x^{0}, \lambda^{0}, \mu^{0}\right)$ is in $\mathrm{Q}$ with $\mu_{j}^{0} \partial g_{j}\left(x^{0}\right)=0$, for all $\mathrm{j}$

$$
\begin{aligned}
& \text { now from }(15) \text {, we have } \\
& \sum_{i=1}^{k} \lambda_{i}^{0} \alpha_{i}+\sum_{j=1}^{m} \mu_{j}^{0} \beta_{j}=0
\end{aligned}
$$

for some for some $\alpha_{i} \in \partial f_{i}\left(x^{0}\right)$ for all $i$ and $\beta_{\mathrm{i}} \in \partial g_{\mathrm{j}}\left(x^{0}\right)$ for all $\mathrm{j}$.

Now , by the feeble duality theorem 3 , we can conclude that $\left(x^{0}, \lambda^{0}, \mu^{0}\right)$ is an well-organized answer for (D).

Assume that $\left(x^{0}, \lambda^{0}, \mu^{0}\right) \quad\left(x^{0}, \lambda^{0}, \mu^{0}\right)$ is not a correctly well-organized answer for (D). Then, there exists a point 
$(u, \lambda, \mu) \in Q$ and an index i such that $f_{i}(u)-f_{i}\left(x^{0}\right)$ can be made arbitrarly large and hence $\lambda>0$ with $\lambda^{t} e=1$

and $b\left(x^{0}, u\right)>0$, we get

$$
\sum_{i=1}^{k} b_{i}\left(x^{0}, u\right) \lambda_{i} f_{i}\left(x^{0}\right)<\sum_{i=1}^{k} b_{i}\left(x^{0}, u\right) \lambda_{i} f_{i}(u)
$$

by the $V-(b, F, \rho)$-pseudoconvexity of $\lambda f$ and the insurable of $\mathrm{F}$, we have

$$
F\left(x^{0}, u_{i} \sum_{i=1}^{k} \lambda_{i} \alpha_{i}+\left(\sum_{i=1}^{k} \rho_{i}\right) d^{2}\left(x^{0}, u\right)<0\right.
$$

Now since $x^{0} \in S \quad\left(u_{x} \lambda, \mu\right) \in Q$ and by the $V-\left(c_{x} F_{x} \gamma\right)-$ quasi convex at $\mu g$ and the insurable of $\mathrm{F}$, we have

$$
F\left(x^{0}{ }_{w} u_{i} \sum_{j=1}^{\mathrm{m}} \mu_{j} \beta_{j}+C \sum_{j=1}^{\mathrm{m}} \gamma_{j}\right) d^{2}\left(x^{0}{ }_{a} u\right) \leq 0
$$

Now from (17),(18) and (19) and (11) and by insurable of F , we get

$$
F\left(x^{0}, u_{i} \sum_{i=1}^{k} \lambda_{i} \alpha_{i}+\sum_{j=1}^{m} \mu_{j} \beta_{j}\right)<0
$$

Which contradicts the fact $F\left(x^{0}, u_{0}, 0\right)=0$. Thus $\left(x^{0}, \lambda^{0}, \mu^{0}\right)$ is a correctly well-organized solution for (D).

Theorem 5.3 (Strict reverse Duality) Let $x^{0} \in S$ and $\left(u^{0}, \lambda^{0}, \mu^{0}\right) \in Q$ such that

$$
f\left(x^{0}\right)=f\left(u^{0}\right) \quad \text { (19) }
$$

If $\lambda^{0} f$ is V-strictly $-(b, F, \rho)$-pseudoconvex at $\mu^{0}$ and $\mu^{0} g$ is $V-(c, F, \gamma)$ - quasi convex at $\mu^{0}$ such that (11) is satisfied then $x^{0}=u^{0}$.

Proof: Suppose that $x^{0} \neq u^{0}$. Since $\left(u^{0}, \lambda^{0}, \mu^{0}\right) \in Q$ and the insurable of $\mathrm{F}$, we have

$$
F\left(x^{0}, u^{0}{ }_{s} \sum_{i=1}^{k} \lambda_{i}^{0} \alpha_{i}+\sum_{j=1}^{m} \mu_{j}^{0} \beta_{j}=0\right.
$$

for some for some $\alpha_{i} \in \partial f_{i}\left(x^{0}\right)$ for all $i$ and $\beta_{\mathrm{i}} \in \partial g_{\mathrm{j}}\left(x^{0}\right)$ for all $\mathrm{j}$.

Since $c\left(x^{0}, u^{0}\right) \geq 0, x^{0} \in S$ and $\left(u^{0}, \lambda^{0}, \mu^{0}\right) \in Q$ and by $V-(c, F, \gamma)$ quasi convexity of $\mu^{0} g$ and by the insurable of $F_{\text {a we have }}$

$$
F\left(x^{0}, u^{0_{0}} \sum_{j=1}^{m} \mu_{j}^{0} \beta_{j}+\left(\sum_{j=1}^{m} \gamma_{j}\right) d^{2}\left(x^{0}{ }_{w} u^{0}\right) \leq 0\right.
$$

now, from (19) and since $\lambda^{0} f$ is V-strictly $-(b, F, \rho)$-pseudoconvex at $\mu^{0}$ we get

$$
F\left(x^{0}, u^{0}{ }_{s} \sum_{i=1}^{k} \lambda_{i}^{0} \alpha_{i}+\left(\sum_{i=1}^{k} \rho_{i}\right) d^{2}\left(x^{0}, u^{0}\right)<0\right.
$$

Now from (11), (21) and (22) and by the insurable of F, $F\left(x^{0}, u^{0_{0}} \sum_{i=1}^{k} \lambda_{i}^{0} \alpha_{i}+\sum_{j=1}^{m} \mu_{j}^{0} \beta_{j}\right)<0$

which contradicts (20) Thus $x^{0}=u^{0}$.

\section{REFERENCES}

1. F.H.Clarke, , optimization and nonsmooth analysis, Wiley,New York . 1983

2. R.R.Egudo, and M.A. Hanson, FSU Technical report Number M-888, 1993.

3. A.M.Geoffrion,., Journal of Mathematical Analysis and Applications, 22,618-630, 1968.

4. M.A.Hanson, .and B.Mond,.,Journal of Information and Optimization Sciences, 3,25-32. 1982.

5. V.Jeyakumar, and B.Mond.., Journal of Australian Muthematical Society (Series B), 39,45-53 1996.

6. C.S.Lalitha,., International Journal of management and System, 11,183-198 1995 .

7. O.L.Mangasarian, , Nonliinear programming, McGraw Hil.New York ,1969.

8. S.K.Mishra . and R.N. Mukherjee., Journal of Australian Muthematical Society (Series B), 38,140-148, 1996.

9. P.Pandian. ANJAC J.sci., I 46-56, 2002.

\section{AUTHORS PROFILE}

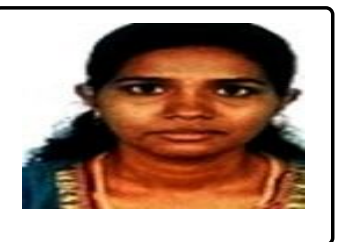

P. Jeyalakshmi pursued her B.sc degree from Madurai Kamarajar University, Madurai in 1999. She got her M.Sc from Madurai Kamarajar University, Madurai in 2001 and her M.Phil degree from Madurai Kamarajar University, Madurai in 2002 She had 10 years of teaching experience. Now, she is doing research in Kalasalingam Academy Of Research And Education, Anand Nagar, Krishnankovil-626126, Tamilnadu, India. 